\title{
Gauge fixing and Gribov copies in pure Yang-Mills on a circle.
}

\author{
James E. Hetrick ${ }^{\mathrm{a}}$

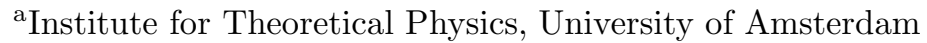 \\ Valckenierstraat 65, 1018 XE Amsterdam, The Netherlands
}

\begin{abstract}
In order to understand how gauge fixing can be affected on the lattice, we first study a simple model of pure Yang-mills theory on a cylindrical spacetime $\left[S U(N)\right.$ on $\left.S^{1} \times \mathbf{R}\right]$ where the gauge fixed space is explicitly displayed. On the way, we find that different gauge fixing procedures lead to different Hamiltonians and spectra, which however coincide under a shift of states. The lattice version of the model is then compared.
\end{abstract}

\section{Introduction}

A crucial question for understanding lattice gauge fixing, is how the reduction of a gauge theory [1] is carried over from the continuum to the discretized version by the Wilson formulation. Given a theoretical understanding of this processs, efficient algoritms can be explored.

The analysis here continues a series of steps [2,3] in understanding the topological structures involved and their remains upon discretization. Here we present the gauge fixing of a simple model where the above steps can be explicitly displayed analytically.

For $S U(N)$ gauge fields in $1+1$ dimensions where space is compactified to a circle [4], the spectrum is discrete and depends on the choice of gauge fixing.

In general, two dimensional Yang-Mills theory on a Riemann surface is a topological theory, whose analysis is presently the subject of much research [7].10]. These theories have only nonperturbative excitations which depend on the underlying topology of their spacetime. In two dimensions, Yang-Mills field theory becomes a finite dimensional quantum mechanics of the eigenvalues of the holonomies (Wilson loops) around each non-contractible circle. The addition of fermions has an interesting effect on the vacuum structure as pointed out in 5.9 .17 , however we will confine ourselves to the pure gauge case in this talk for the purpose of displaying the gauge fixing constructs.

The fundamental fields of the theory are the gauge fields $A_{\mu}(x, t)$ defining the coordinates of the unreduced configuration space, with the usual Lagrangian,

$\mathcal{L}=-\frac{1}{2} \operatorname{Tr} F_{\mu \nu} F^{\mu \nu}$,

The fields $A_{\mu}$ and the curvature $F_{\mu \nu}$ take their values in the Lie algebra $\widehat{g}$ of $G=S U(N)$. On the circle we can, without loss of generality, take $A_{\mu}(x+L, t)=A_{\mu}(x, t)$.

The canonical momenta to the $A_{\mu}$ are:

$\Pi_{\mu}^{a}=E_{\mu}^{a}=\frac{\delta \mathcal{L}}{\delta \partial_{0} A_{\mu}^{a}}$,

and we see immediately that $\Pi_{0}$ vanishes identically.

The classical Hamiltonian is then

$H=\frac{1}{2} \int_{0}^{L} d x\left\{E_{1}^{2}+2 A_{0} D_{1} E_{1}\right\}$

and $A_{0}$ emerges as a Lagrange multiplier field for the constraint of Gauss' law

$D_{1} E_{1} \sim 0$

which must vanish on the reduced phase space $(\sim$ $0)$. Thus we can set $A_{0}=0$ in further discussion.

As a secondary constraint, $D_{1} E_{1}$ generates gauge transformations on the partially reduced phase space [1] which we must reduce further to the equivalence classes under gauge transformations.

\section{Gauge Fixing}

The configuration space of the theory is now $\mathcal{A}=\left\{A_{1}(x) \mid A_{1}: S^{1} \mapsto \widehat{g}\right\}$, the set of maps 
of the circle into $\widehat{g}$. Similarly the space of gauge transformations is the set of maps from the circle into the gauge group, $\mathcal{G}=\left\{\Lambda(x) \mid \Lambda: S^{1} \mapsto\right.$ $G\}$. The true configuration phase space is then formally

$\mathcal{A} / \mathcal{G}$.

What makes this circle model so convenient is the fact that $\mathcal{A}$ and $\mathcal{G}$ have been well studied 11. $\mathcal{G}$ is the loop group of $G(\equiv L G)$, whose definition is given above, and $\mathcal{A}$ is isomorphic to the tangent space of $L G(\equiv L \widehat{g})$.

$L G$ has the following structure:

$L G(=\mathcal{G}) / G \equiv \Omega G$

where $\Omega G$ is the set of based loops in $G$, that is, the set of maps $\left\{\Lambda: S^{1} \mapsto G\right\}$ such that $x=0$ ( $\epsilon$ $\left.S^{1}\right) \rightarrow \mathbf{1}(\in G)$.

It is known that 6, 11, 12

$L \widehat{g}(=\mathcal{A}) / \Omega G \cong G$.

Then, using eqs. (6) and (7) in (5) gives:

$\mathcal{A} / \mathcal{G} \cong G / G$.

where $G / G$ is obtained by identifying the points $x^{\prime}$ and $x \in G$ which are conjugate: $x^{\prime}=a^{\dagger} x a, \forall a$, under the action of $G$ on itself. This space is an orbifold made by identifying points in the maximal Abelian subgroup of $G$ (the maximal torus $T_{G}$ ), under the action of the Weyl group $W_{G}$, a discrete set of transformations which shifts an element of $T_{G}$ by the center $Z_{N}$, or permutes its diagonal elements. Thus

$\mathcal{A} / \mathcal{G} \cong T_{G} /\left(Z_{N} \times S_{N}\right)$,

and we have an exact identification of the physical configuration space of the theory as the orbifold $T_{S U(N)} /\left(Z_{N} \times S_{N}\right)$.

\subsection{Coulomb Gauge}

The above analysis gives the topology of the gauge orbit space. How do we see this structure emerge in the more colloquial method of gauge fixing, say to Landau or Coulomb gauge conditions?

In the Coulomb gauge [5, 9] for example, we must find a gauge transformation $\Lambda(x, t)$ such that

$\partial_{1}\left\{\Lambda A_{1} \Lambda^{\dagger}+i g \Lambda \partial_{1} \Lambda^{\dagger}\right\}=0, \quad \forall A_{1}(x, t)$.
Eq. (10) is solved by taking

$\Lambda=e^{-i B x / L} \exp \left\{\int_{0}^{x} d y A_{1}(y, t)\right\}$

where

$B=B(t)=\int_{0}^{L} d y A_{1}(y, t)$

is the zero momentum mode of $A_{1} \cdot \exp \{-i B(t)\}$ is the holonomy around the circle. Furthermore, within the Coulomb gauge condition we have the freedom to make $x$-independent gauge transformations, and so we can diagonalize $A_{1}(t)$ on each time slice of the cylinder:

$A_{1}(t) \rightarrow \frac{1}{g L}\left(\begin{array}{cc}\theta_{1}(t) & \\ & \ddots \\ & \theta_{N}(t)\end{array}\right) \equiv \Theta(t)$.

As $A_{1}$ is traceless, $\sum_{i=1}^{N} \theta_{i}(t)=0$. In this complete Coulomb gauge one can easily show that $A_{0}$ decouples [5].

Now we see the physical configuration space emerging, since $\Theta$ is an $N-1$ dimensional flat sub-manifold of $\mathcal{A}$, which will become the maximal torus $T_{S U(N)}$ upon the identification Gribov copies.

\subsection{Gribov Copies}

Notice that maintaining the periodicity of $A_{\mu}(x)$ around the circle, requires $\Lambda$ to satisfy:

$\Lambda(x+L, t) \Lambda^{\dagger}(x, t)=Z_{N}$

where $Z_{N}$ is in the center of $S U(N)$. Thus eq. (13) is preserved under gauge transformations

$\Lambda(x)_{j k}=\exp \left\{\frac{2 \pi i x}{L}\left(n_{j}+m\right)\right\} \delta_{j k}$

where $n_{j}$ and $m$ satisfy

$$
\begin{gathered}
n_{j} \in \mathbb{Z}, \quad \sum_{j=1}^{N} n_{j}=0 \\
m=\kappa / N, \quad \kappa=1, \ldots, N-1
\end{gathered}
$$

such that the action on $\Theta$ is

$\theta_{j}(t) \rightarrow \theta_{j}(t)+2 \pi n_{j}+2 \pi m$.

The identification of the points $n_{j}$ in the space spanned by $\Theta$ make it isomorphic to the maximal 
torus $T_{S U(N)}$, while the Gribov copies labeled by $m$ make the $Z_{N}$ identifications. Similarly, a further set of Gribov copies $\Lambda(t)$ permutes the $\theta_{j}(t)$. These then, are all the residual Gribov copies left after completely fixing to Coulomb gauge. Thus the identification of these copies gives the linear subspace $\Theta$, coming from Coulomb gauge fixing, the topology of the physical configuration space, $T_{S U(N)} /\left(Z_{N} \times S_{N}\right)$.

The Gribov copies are essential to identifying the correct topology of the configuration space which manifests, for instance, in the periodicity of the wave function $\Psi\left(\theta_{j}\right)$. This argument has also been stressed in [13] and [17].

\section{Quantization}

The Hamiltonian, on the unreduced configuration space, must be projected down onto the gauge fixed surface (via Dirac brackets or geometric methods). In the case at hand,

$H=\int_{0}^{L} d x \Pi_{1}^{2}=\int_{0}^{L} d x \frac{\delta^{2}}{\delta A_{1}^{2}(x)}$

and there are at least two projections in the literature.

The method in [5] and [9] is to take the restriction of eq. (19) to the hyperplane in $\mathcal{A}$ spanned by $\Theta$, then impose the periodicity of the Gribov copies on the wave funtions. For $S U(2)$,

$$
\begin{gathered}
H=\frac{g^{2} L}{8} \frac{\partial^{2}}{\partial \theta^{2}} \\
\Psi(\theta)=\cos (n \theta), \quad E_{n}=g^{2} L n^{2} / 8 ; \quad n \in \mathbb{Z}_{\geq 0}
\end{gathered}
$$

In [4] and [6], the full Hamiltonian in eq. (19) is projected first onto the subspace of $\mathcal{A}$ isomorphic to $G$, identified there with the Laplacian on $G$, and the equivalence of conjugate $(G / G)$ configurations is imposed on the wave functions. Since $H=\Delta_{S U(N)}$, wave functions are the characters of $S U(N)$ and the spectrum is the quadratic Casimir. Thus for $S U(2)$ again:

$$
\begin{aligned}
& \Psi(\theta)=\sin \left(\left[j+\frac{1}{2}\right] \theta\right) / \sin (\theta / 2) \\
E_{n}= & g^{2} L j(j+1) / 2 \quad j \in \mathbb{Z}_{\geq 0} / 2 .
\end{aligned}
$$

It would seem that these spectra are completely different, however notice that adding $\frac{g^{2} L}{8}$ to the
Casimir spectrum reproduces the spectrum of eq. 8 , up to the disappearance of the ground state with $E_{n=0}=0$ since

$j(j+1) / 2+1 / 8=n^{2} / 8 ; \quad \forall n \in \mathbb{Z}_{>0}, j \in \frac{\mathbb{Z}_{\geq 0}}{2}(24)$

Similarly, for $S U(3)$, the quantization of a particle on the maximal torus $T_{S U(3)}$ gives [5.9]:

$E_{\lambda, \mu}=\frac{g^{2} L}{6}\left(\lambda^{2}+\lambda \mu+\mu^{2}\right) ; \quad \lambda, \mu \in \mathbb{Z}$

Taking $\lambda \rightarrow \lambda+1, \mu \rightarrow \mu+1$ yields $c_{2} / 2+1 / 2$, where the quadratic Casimir of $S U(3)$ is $c_{2}=$ $\left(\lambda^{2}+\lambda \mu+\mu^{2}\right) / 3+\lambda+\mu$.

The addition of certain constants (and higher Casimirs) to the spectrum has also been noted by Witten [8] and appears to be related to the relationship of 2-D Yang-Mills to the equivalent topological $B F$ theory. Here the added constant is one half the scalar curvature of $G$, coming from the relation between the Hamiltonians on $G$ and $T_{S U(N)}$ [18].

The disappearance of the ground state wave function $\Psi_{n=0}$ in the identification of the spectra is due to the identification of wave functions from $G$ to $T_{S U(N)}$, which involves the metric of $G$ [19]. This becomes non-trivial when fermions are included [5, 17].

\section{Lattice Version}

In two dimensions the lattice partition function $Z$ of pure Yang-Mills theory factorizes into "pant diagrams" which can be glued together by integrating over the holonomy $W$ at the common boundary [7,14 16] as shown in figure 1. After integrating out all internal links, the contribution of the pant diagram $Z_{1}\left(A_{1} ; W_{a}, W_{b}, W\right)$ to $Z$ is

$$
Z_{1}=\sum_{r} d_{r}^{-1} \lambda_{r}^{A_{1} / a^{2}} \chi_{r}\left(W_{a}\right) \chi_{r}\left(W_{b}\right) \chi_{r}(W)
$$

where $r$ runs over representations of $G$, of dimension $d_{r} ; \chi_{r}\left(W_{i}\right)$ is the character of the Wilson loop $W_{i}$ in representation $r, A_{1}$ is the area of the Riemann surface $Z_{1}$, and $a$ is the lattice spacing. $\lambda_{r}$ is the Fourier component of the character expansion of the lattice action

$$
\lambda_{r}=d_{r}^{-1} \int d U_{\square} \chi_{r}\left(U_{\mathbf{\square}}\right) \exp \left\{\beta_{0} N \operatorname{Tr} U_{\mathbf{\square}}\right\} .
$$


Figure 1. The partition function with external states $W_{a}, W_{b}, W_{c}$, and $W_{d}$ : $Z_{1+2}=$ $\int d W Z_{1}\left(W_{a}, W_{b}, W^{\dagger}\right) Z_{2}\left(W, W_{c}, W_{d}\right)$

With the $n$-point pant diagrams one can analytically calculate the expectation value of a Wilson loop in the representation $R$, at the seam $W$, by inserting an extra character $\chi_{R}(W)$ into the computation of the partition function $Z_{1+2}$, in figure 1.

To see which of the above spectra the nongauge-fixed lattice choses, we compute

$$
<W(t) W(0)>=\sum_{n}|<0| \widehat{W}|n>|^{2} e^{-t E_{n}}
$$

by gluing 3 cylinders in series, with Wilson loop characters sandwiched in between, and setting $W_{\text {ends }}=\mathbf{1}$. Taking the areas of the external cylinders very large, one finds that $\langle W(t) W(0)\rangle \sim$ $\exp \left(-c_{2} t / 2\right)$ in accordance with the Casimir spectrum of eq. (23). This is, of course, not surprising since the lattice formulation is compact, hence the quantization is done on $G$ as in eq. (23). Similarly, one can use a Hamiltonian approach, with $A_{0}=0$, finding again a Casimir spectrum, for the same reasons of compactness.

It appears that the lattice (or quantization on the group manifold) cannot see the true ground state of this model, that of a constant wave function on the gauge orbit space $T_{S U(N)} /\left(Z_{N} \times S_{N}\right)$. The lattice ground state is a constant $\Psi_{j=0}$ on $G$, which projects to the $\Psi_{n=1}$ state on the maximal torus [19].

\section{Acknowledgements}

I wish to thank many people for stimulating and insightful disscussions on the above topics, in particular: Y. Hosotani, J. Smit, Ph. de Forcrand, R. Dijkgraaf, P. van Baal, D. Birmingham, F.A. Bais, and M. Blau.

\section{REFERENCES}

1. D. M. Gitman and I. V. Tyutin, Quantization of Fields with Constraints, Springer-Verlag (1990)

2. Ph. de Forcrand et al., Nucl. Phys. B (Proc. Suppl.) 20 (1991), 194

3. J. E. Hetrick et al., Nucl. Phys. B (Proc. Suppl.) 26 (1992), 432

4. S. G. Rajeev, Phys. Lett. B212 (1988), 203

5. J. E. Hetrick and Y. Hosotani, Phys. Lett. B230 (1989), 88

6. J. Mickelsson, Phys. Lett. B242 (1990), 217

7. E. Witten, Comm. Math. Phys. 141 (1991), 153

8. E. Witten, IASSNS-HEP-92/15 "Two Dimensional Gauge Theories Revisited" hepth/9204083

9. E. Langmann and G. W. Semenoff, Univ. British Columbia preprint UBCTP 92019 "Gauge theories on a cylinder" hepth/9210011

10. D. Birmingham et al., Phys. Rep 209 (1991), 129

11. A. Pressley and G. Segel, Loop Groups, Oxford (1986)

12. M. F. Atiyah and R. Bott, Philos. Trans. R. Soc. Lond., 308A (1982), 523

13. P. van Baal, Nucl. Phys. B369 (1992), 259

14. B. Ye. Rusakov, Mod. Phys. Lett. A5 (1990), 693

15. J. F. Wheater, Phys. Lett. B264 (1991), 161

16. M. Blau and G. Thompson, Int. J. Mod. Phys. A7 (1992), 3781

17. E. Langmann and G. W. Semenoff, "Gribov ambiguity and non-trivial vacuum structure of gauge theories on a cylinder" hep-th/9212038

18. J. S. Dowker, Ann. Phys. 62 (1971), 361

19. J. E. Hetrick, hep-lat/9305020 